\title{
ANÁLISE DE DESEMPENHO DE SENSORES PARA TRANSMISSÃO DE VÍDEO EM REDES SEM FIO DE BAIXA VAZÃO
}

\author{
Marcelo Bião Cerqueira ${ }^{1}$; Daniel Gouveia Costa ${ }^{2}$ \\ 1. Bolsista PIBIC/CNPq, Graduando em Engenharia de Computação, Universidade Estadual de Feira de Santana, e- \\ mail: marcelobiao2@gmail.com \\ 2. Orientador, Departamento de Tecnologia, Universidade Estadual de Feira de Santana, e-mail: \\ danielgcosta@uefs.br
}

PALAVRAS-CHAVE: Redes de Sensores Sem Fio, Redes Multimídia, Codecs.

\section{INTRODUÇÃO}

As Wireless sensor networks(WSN) ou rede de sensores sem fio são definidas como uma distribuição espacial de sensores autônomos para monitoramento de condições físicas ou ambientais. As WSN são habitualmente utilizadas em ambientes com baixa infraestrutura, pois desprezam a presença de fios, devido à natureza de sua comunicação e a utilização de baterias para alimentação dos módulos, essas aplicações são frequentemente utilizadas para na aquisição de informações de plantações, industrias, veículos automotores entre outras.

Alguns tipos de redes necessitam lidar com uma maior carga de dados para processar devido à natureza dos seus sensores, esse é o caso das Wireless Visual Sensor Network, ou Rede De Sensores Visuais Sem Fio (RSVSF), composta por sensores multimídia. A utilização de sensores multimídia geram dois grandes problemas, primeiro o custo associado a aquisição, pois trata-se de sensores complexos com alto custo de fabricação e segundo o custo associado ao transporte e processamento do grande volume de dados que esses sensores geram. Uma forma de contornar esse problema é através da codificação dos dados.

O objetivo da codificação é reduzir o volume de dados, através da representação das imagens ou vídeos com o menor número possível de bits, sem a alteração da qualidade e da nitidez necessária a aplicação. A codificação realiza uma compressão na imagem, facilitando sua transmissão e armazenamento.

A compressão de uma imagem só é possível porque as imagens apresentam um alto graus de coerência, que se traduz em uma redundância de informação quando codificada. A compressão de imagens pode ocorrer de duas maneiras possíveis, com e sem perda de informação. A Compressão sem perda ou codificação de redundância, explora a redundância entre pixels na codificação, preservando todas as informações que permitirão a reconstrução exata da imagem. A compressão com perda gera uma degradação de dados durante o processo de compressão da imagem, sendo mais eficiente em relação à área final de armazenamento devido à sua razão de compressão ser maior que a sem perda.

Outra estratégia para contornar o alto fluxo de dados e custo de implementação da rede é a utilização de sensores da nova geração, que possuem maior capacidade de processamento e armazenamento de dados, e cujos custos de aquisição vem caindo ao longo do tempo. O Single Computer Board, Raspberry P $i$, está entre os potenciais dispositivos de baixo custo que podem atuar como nó sensor e ao longo dos anos vem se tornando popular, por ser um dispositivo barato, de baixo consumo e com inúmeras interfaces de entrada e saída. 


\section{MATERIAL E MÉTODOS OU METODOLOGIA (ou equivalente)}

A primeira etapa dos testes, foi a avaliação dos Sistemas Operacionais que serão utilizados neste trabalho. Para essa avaliação, eles foram instalados no módulo Raspberry PI, apenas com o essencial para o seu funcionamento. Durante a avaliação foi observado alguns critérios fundamentais para um Sistema Operacional, apresentados a seguir:

- Portabilidade: O tamanho da imagem do Sistema Operacional dita a capacidade que o cartão SD deverá ter. Imagens maiores necessitam de mais espaço, restringindo diretamente a quantidade de dados que um nó sensor possa armazenar internamente, além de aumentar o custo com cartão maiores.

- Consumo de memória em repouso: Mais importante que que o espaço ocupado no cartão SD é o consumo de memória RAM, já que o Chip de memória de Raspberry não podem ser substituídos, limitando as aplicações $1 \mathrm{~GB}$.

- Uso do processador em repouso: Esta característica indica a quantidade de serviços que executam para manter o $\mathrm{SO}$ em funcionamento. Mais serviços indicam mais processamento e o uso da CPU está diretamente ligada com o consumo energético.

- Tempo de boot: É possível combinar o uso do Raspberry Pi com microcontroladores que consomem menos energia. Os microcontroladores seriam responsáveis por monitorar a rede, acordando o Raspberry Pi apenas para realizar tarefas mais complexas. Saber o tempo de boot é importante pois a informação pode perder relevância com o passar do tempo.

- Customização: É interessante que o SO tenha a capacidade de ser modificado para atender necessidades específicas, desativando componentes ou incluindo serviços novos. Sistemas Open-source são sempre uteis nesses casos.

- Documentação: Uma base solida de informação na comunidade ajuda no desenvolvimento de aplicações para essa plataforma e a contornar problemas ocasionados por bugs, devido ao grande número disponibilizados através de manuais, listas de discursões e documentações oficiais e não oficiais.

Com os Sistemas Operacionais e o modulo da câmera instalados, começou o processo de aquisição das imagens. Primeiro foi adquirido a imagem sem codificação, mapa de bits, e depois a imagem foi codificada para cada um dos codecs avaliados, sendo medido o consumo energético durante esse processo. A codificação consiste em eliminar pixels redundantes para economizar espaço de armazenamento, sendo que essa eliminação pode ocorrer com ou sem perda de informação.

Métodos para a compactação de dados com perdas, necessitam de uma métrica padronizada para mensurar a qualidade da imagem reconstruída pela compactação, em relação a imagem original. $\mathrm{O}$ valor produzido por essa métrica deve ser adimensional e não deve sofrer variações bruscas em decorrência de pequenas variações nas imagens, tendo em vista que quanto mais uma imagem reconstruída se assemelha a original, maior deve ser o valor produzido por essa métrica. 
Uma medida comum usada para esta finalidade é a relação peak signal to noise ratio, ou PSNR. Realizar o cálculo do PSNR é simples, pois o sistema apresenta uma relação simples e aproximada dos erros percebidos do sistema visual humano.

\section{RESULTADOS E/OU DISCUSSÃO (ou Análise e discussão dos resultados)}

As medições de corrente elétrica foram realizadas na entrada de alimentação do Raspberry $P \grave{i}$, que foi alimentado com uma fonte $5 \mathrm{~V}$ DC com $1 \mathrm{~A}$ de corrente máxima. Os valores de potência dos dispositivos apresentados a seguir são expressados em Watt, produto da tensão pela corrente.

Quando se trata de uma aplicação embarcado que tem energia como preocupação, não vale a pena manter módulos do dispositivo em funcionamento sem necessidade. No Raspberry Pi é possível desativar algumas funcionalidades a fim de reduzir o gasto energético do dispositivo, a seguir é demonstrado uma comparação entre os SO com os quesitos estabelecidos na sessão, com os dispositivos em repouso.

\begin{tabular}{|c|c|c|c|}
\hline QuesitolSO & Rpi Jessie & Arch Linux & Raspbian OS \\
\hline Tamanho da Imagem & $1.29 \mathrm{~Gb}$ & $1.86 \mathrm{~Gb}$ & $1.3 \mathrm{~Gb}$ \\
\hline Memória & $177 \mathrm{Mb}$ & $120 \mathrm{Mb}$ & $200 \mathrm{Mb}$ \\
\hline Quantidade de Processos & 103 & 89 & 122 \\
\hline Tempo de boot & $15.7 \mathrm{~s}$ & $8.2 \mathrm{~s}$ & $19.1 \mathrm{~s}$ \\
\hline Customizável & Sim & Sim & Sim \\
\hline Suporte PiCamera & Sim & Sim & Sim \\
\hline Consumo & $1.02 \mathrm{Watt}$ & $0.98 \mathrm{Watt}$ & $1.11 \mathrm{Watt}$ \\
\hline
\end{tabular}

Os scripts para testar os codecs de vídeo foram implementados para ativar o módulo da câmera e realizar takes de 10 segundo com cada Codec e durante esse processo foi realizado a medição do consumo energético do módulo durante a execução dos testes, realizando uma média entre as medições, essa média é exibida a seguir.

\begin{tabular}{|c|c|c|}
\hline Codec & Consumo(Watt) & Tamanho do arquivo \\
\hline MJPEG & 1.29 & $17 \mathrm{Mb}$ \\
\hline H264 & 1.28 & $992 \mathrm{~Kb}$ \\
\hline
\end{tabular}

Com a execução dos testes, notamos que na prática o consumo dos dois codecs é bem similar, sendo o H264 em relação ao armazenamento superior ao MJPEG. A principal diferença entre o H254 e MJPEG é que o MJPEG comprime apenas quadros individuais, enquanto o H264 comprime quadros em sequência, isso possibilita que o H264 salve apenas a alteração entre os quadros, resultando em um vídeo mais compacto, porém com uma menor qualidade em relação ao MJPEG. 
Os scripts para análise dos codecs de imagem, foram implementados para tirar 10 imagens em sequência com cada codec e foi realizado a medição do consumo energético do módulo durante a execução dos testes. A seguir é apresentado um comparativo entre os codecs com os seguintes critérios: Tamanho, representa o tamanho do arquivo de imagem de uma das amostras colhidas; tempo, representa o tempo de execução do script de teste; Consumo, representa gasto energético médio durante a execução do script; e PSNR5, representa o grau de similaridade entre as imagens, quando comparadas com o mapa de bits (sem codificação).

\begin{tabular}{|c|c|c|c|c|}
\hline Formato & Tamanho & Tempo(s) & Consumo(Watt) & PSNR5 \\
\hline GIF & $470 \mathrm{~Kb}$ & $13 \mathrm{~s}$ & 1,25 & 41.72 \\
\hline PNG & $1,3 \mathrm{Mb}$ & 19,5 & 1.22 & 46.20 \\
\hline JPG & $614 \mathrm{~Kb}$ & $6,5 \mathrm{~s}$ & 1,27 & 43.89 \\
\hline BMP & $3 \mathrm{Mb}$ & $13 \mathrm{~s}$ & 1,28 & $\infty$ \\
\hline
\end{tabular}

Maior semelhança entre as imagens implica em um maior PSNR. Vale lembrar que maiores valores de PSNR implicam em uma menor discrepância entre as imagens reconstruídas e originais, porém não garante que os observadores gostem do resultado.

\section{CONSIDERAÇÕES FINAIS (ou Conclusão)}

Durante a execução dos testes, é possível notar que as execuções dos diferentes algoritmos de codificação, tanto de imagem e vídeo, geram prejuízos diferentes, em relação ao tamanho dos arquivos gerados e consumo energético, mas também geram benefícios, como uma melhor qualidade das imagens e vídeos obtidos dos nós sensores da rede.

Em uma aplicação, deve primeiro analisar qual a sua principal preocupação. Caso seja emergir, optar por algoritmos mais econômicos energeticamente em detrimento da qualidade das imagens é necessário para garantir uma maior vida útil das baterias que alimentam os nós sensores, como é o caso do JPG. Para aplicações mais robustas, onde a energia para alimentar os módulos não é a preocupação, ou a necessidade de uma melhor resolução dos dados colhidos é necessário, podemos optar por algoritmos, que irão gerar uma melhor qualidade dos dados como é o caso do MJPEG e PNG. Ou seja, detectar as necessidades da sua aplicação, é fundamental para a escolha dos métodos utilizados para obter uma melhor otimização do sistema final.

\section{REFERÊNCIAS}

[1] R. P. Foundation. Raspberry pi camera. http://picamera.readthedocs.io/en/release-

1.13/. Acessada em: 04/06/2017.

[2] D. Salomon. Data Compression. Springer, 4th edition, 2007.

[3] H. ZainEldin. Image compression algorithms in wireless multimedia sensor networks: A survey. Ain Shams Engineering Journal, 2015.

[4]CALIN, D. The Raspberry Pi camera guide. 2015. Disponível em: https://www.intorobotics.com/raspberry-pi-camera-guide/ . Acesso em: 12 abr. de 2016. [5]MAMMERI, A.; HADJOU, B.; KHOUMSI, A. A survey of image compression algorithms for visual sensor networks. ISRN Sensor Networks, Hindawi Publishing Corporation, v. 2012, 2012. 